\title{
Cuadernos de Psicología del Deporte
}

\section{Editorial}

\section{EL GIMNASIO Y LA ACADEMIA: PARA UNA FILOSOFÍA DEL DEPORTE}

Enrique E. Corrales

Eg Editorial Invitada

Editorial

Juan González Hernández; Antonio Hernandez Mendo

因 CPD1(2019)

\section{Psicología del Deporte}

Adaptação transcultural do Empowering Disempowering Motivational Climate Questionnaire para a língua portuguesa e análise da invariância em atletas masculinos brasileiros e portugueses

Helder Zimmermann Oliveira,Teresa Silva Dias,Bebiana Catarina Rodrigues Sabino,Cláudia Dias,Nuno CorteReal,António Manuel Fonseca

Empowering Disempowering Motivational Climate Questionnaire in portuguese (Português (Portugal))

Imagen Corporal y Obesidad mediante las Siluetas de Stunkard en Niños y Adolescentes Indios de 8 a 15 Años Sheila Sánchez Castillo,Guillermo Felipe López Sánchez,MD Dilsad Ahmed,Arturo Diaz Suárez

四 Imagen Corporal y Obesidad en jóvenes de la India

Efectos de un programa de intervención sobre el estilo interpersonal de entrenadores de fútbol base Juan José Pulido González,Antonio Merino,David Sánchez-Oliva,Miguel Rodriguez-González,Tomás Garcia-Calvo

Th Entrenamiento del estilo interpersonal en entrenadores de fútbol base

Motor differentiations and cognitive skill in pre-scholar age

Marianna Alesi,Manuel Gómez-López,Antonino Bianco

Individuals differences in motor and cognitive skill in pre-scholars (English)

The use of Polar Coordinates in the analysis of motor interaction in football according to the result. Rómulo Díaz Díaz,juan Manuel Garcia Manso,Teresa Valverde Esteve, Enrique Arriaza Ardiles,Eduardo José Ramos Verde

[G Coordenadas polares en el análisis del fútbol (English)
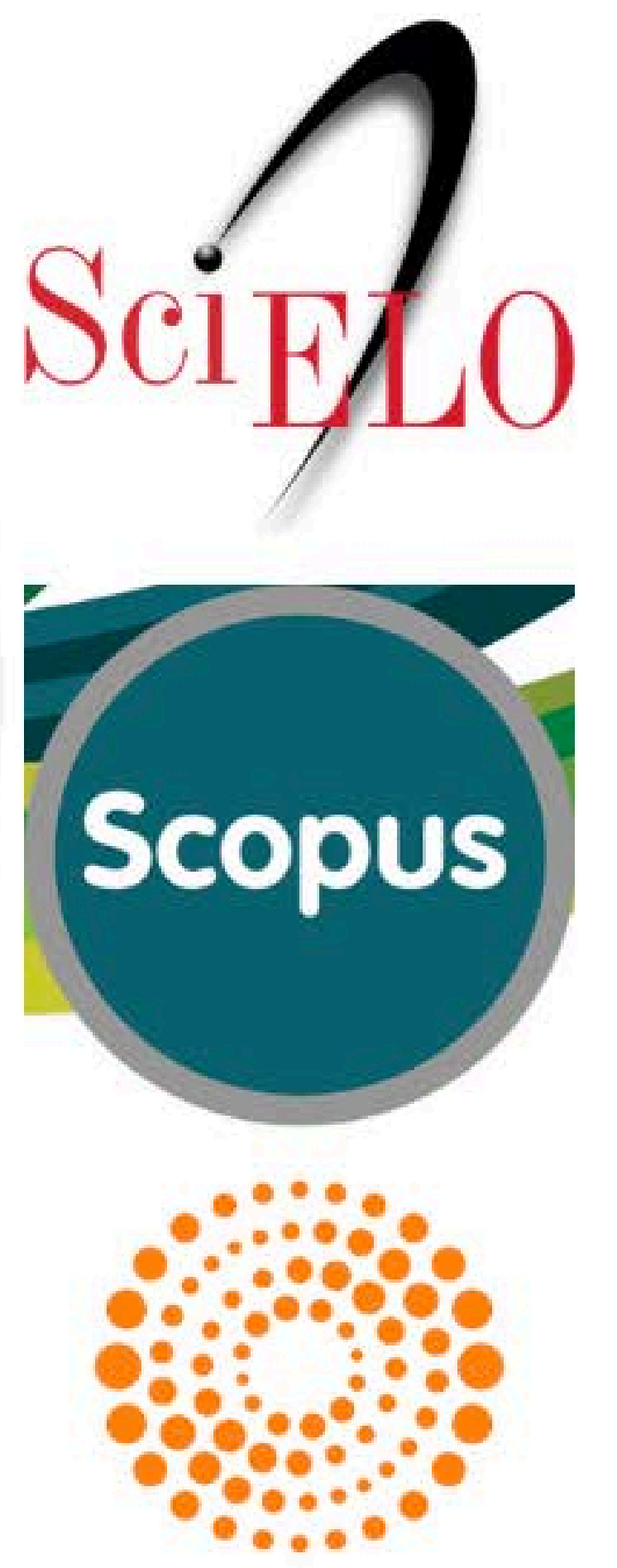

(

\section{THOMSON REUTERS}

INDICES DE IMPACTO CIENTIFICO

WoS. ISI-THOMSON-(pendiente de

asignación indicador JCR)

SCIMAGO SJR 2014: 0.209

Índice H Google Scholar: 10

Primer Cuartil

InRECS FI 2011: 0.633

Primer Cuartil

Cuadernos de Psicologia del Deporte 


\title{
Relación entre clima empowering y diversión en pitchers de béisbol: el papel moderador de la motivación autónoma
}

\section{Relationship between empowering climate and enjoyment in baseball pitchers: the moderator role of autonomous motivation}

\section{Relação entre clima empowering e diversão em arremessadores de beisebol: o papel moderador da motivação autônoma}

\author{
Gutiérrez-García, P. ${ }^{1}$, López-Walle, J. ${ }^{2}$, Tomás, I. ${ }^{3}$, Tristán, J. ${ }^{2}$, y Balaguer, I. ${ }^{3}$ \\ ${ }^{1}$ Universidad Estatal de Sonora. México, ${ }^{2}$ Universidad Autónoma de Nuevo León. México; ${ }^{3}$ Universidad \\ de Valencia. España.
}

\section{RESUMEN}

Tomando como base la conceptualización jerárquica y multidimensional del clima motivacional, propuesto por Duda (2013), el objetivo de este estudio fue poner a prueba el papel moderador de la motivación autónoma en la relación entre la percepción del clima empowering creado por el entrenador y la diversión en la práctica deportiva en pitchers de béisbol. Participaron 97 pitchers Mexicanos $\left(M_{\text {edad }}=14.12, D T=1.09\right)$ pertenecientes a 39 equipos de 10 Ligas de Hermosillo, Sonora, México. Las variables psicológicas fueron evaluadas con las versiones adaptadas al contexto mexicano del Cuestionario de Clima Motivacional Empowering y Disempowering creado por el entrenador (EDMCQ-C), la Escala de Motivación en el Deporte (SMS-II), y la escala de Satisfacción Intrínseca en el Deporte (SSI). Los resultados del modelo de regresión de moderación rodados con PROCESS indicaron que: la motivación autónoma modera la relación entre el clima empowering y la diversión $(\mathrm{B}=-.15, p<.05)$, concretamente, para valores altos de motivación autónoma la relación entre clima empowering y diversión fue nula, pasando a ser esta relación positiva y significativa para valores de motivación autónoma inferiores a 2.05. En conclusión, la motivación autónoma modera la relación entre la percepción del clima empowering creado por el entrenador y la diversión en la práctica de pitchers de béisbol.

Palabras clave: Clima empowering, diversión, motivación autónoma, pitcher de béisbol.

\section{ABSTRACT}

Based on Duda's (2013) hierarchical and multidimensional conceptualization of the motivational climate, the objective of this study was to test the moderating role of autonomous motivation in the relationship between the perception of the empowering climate created by the coach and the enjoyment in the practice by baseball pitchers. Participants were ninety-seven Mexican pitchers $\left(M_{\text {age }}=14.12, S D=1.09\right)$ belonging to 39 teams from 10 Leagues in Hermosillo, Sonora, Mexico. The psychological variables were measured with the adapted to the Mexican context versions of the Empowering and Disempowering Motivational Climate Questionnaire-Coach (EDMCQ-C), the Sport Motivation Scale (SMS-II), and the Sport Satisfaction Instrument (SSI). The results of the moderated regression model with PROCESS indicated that: autonomous motivation moderates the relationship between the

Correspondence to: Jeanette M. López-Walle. Dirección Postal: Av. Universidad s/n, Cd. Universitaria. C.P. 66455. San Nicolás de los Garza, Nuevo León, México. Tel: +52 8113404450. Email: jeanette.lopezw@uanl.edu.mx 


\section{Clima empowering, motivación autónoma y diversión en pitchers de béisbol}

empowering climate and enjoyment $(\mathrm{B}=-.15, p<.05)$, specifically, for high values of autonomous motivation the relationship between climate empowering and fun was null, becoming this positive and significant relationship for values of autonomous motivation lower than 2.05. In conclusion, the autonomous motivation moderates the relationship between the empowering climate perception created by the coach and the enjoyment in the practice of baseball pitchers.

Keywords: Empowering climate, enjoyment, autonomous motivation, controlled motivation, baseball pitcher.

\section{RESUMO}

Baseando-se no conceito hierárquico e multidimensional do clima motivacional, proposto por Duda (2013), o objetivo deste estudo foi colocar em prova o papel moderador da motivação autônoma na relação entre a percepção do clima empowering criado pelo treinador e pela diversão na prática esportiva em pitchers de beisebol. Participaram 97 pitchers mexicanos $\left(M_{\text {idade }}=14.12, D T=1.09\right)$ pertencentes a 39 equipes de 10 ligas de Hermosillo, Sonora, Ciudad de México. As variáveis psicológicas foram avaliadas com as versões adaptadas ao contexto mexicano do Questionário de Clima Motivacional Empowering e Disempowering criado pelo treinador (EDMCQ-C), a Escala de Motivação no Esporte (SMS-II), e a Escala de Satisfação Intrínseca no Esporte (SSI). Os resultados do modelo de regressão de moderação rodados com PROCESS indicaram que: a motivação autônoma condiciona a relação entre o clima empowering e a diversão $(\mathrm{B}=-.15, p<.05)$, concretamente, para altos valores de motivação autônoma a relação entre o clima empowering e diversão foi nula, passando a ser positiva e negativa para valores de motivação autônoma inferiores a 2.05. Concluindo, a motivação autônoma condiciona a relação entre a percepção do clima empowering criado pelo treinador e a diversão na prática de pitchers de beisebol.

Palavras chave Clima empowering, diversão, motivação autônoma, pitcher de beisebol.

\section{INTRODUCCIÓN}

En el ámbito deportivo, el béisbol es uno de los deportes colectivos en el que las acciones tienen lugar en condiciones de interrelación e interdependencia, y además todas las posiciones del juego tienen sus propias particularidades y demandas (Ríos, 2010). Su carácter interdependiente, y sobre todo la individualidad de las posiciones del juego, hacen que sea interesante analizar la relación entre el entrenador y los jugadores que participan en un puesto específico. En el béisbol, el pitcher juega la posición defensiva más complicada del juego en el equipo; ganar el partido depende en gran medida del rol de este importante jugador y de su desempeño en el campo (Ealo, 1984; González, Gotera, y Cobos, 2009). En la literatura, en deportes colectivos está documentado el papel mediador de la motivación en la relación entre los climas motivacionales y los indicadores de bienestar (p.e., Barbosa-Luna, Tristán, Tomás, González, y López-Walle, 2017), así como el papel moderador de la motivación autónoma entre el clima empowering y el burnout (López-Walle et al., 2018). Sin embargo, esta relación no se ha estudiado todavía en el contexto del béisbol, y por lo tanto, nada se conoce de la misma en la posición más determinante del juego, los pitchers.
Por ello, el presente trabajo se centra en analizar el papel modulador que la motivación autónoma juega en la relación entre la percepción de los pitchers de béisbol del clima motivacional de empoderamiento (clima empowering) creado por el entrenador y la diversión en su práctica deportiva.

La investigación ha informado de forma consistente que los climas motivacionales creados por los entrenadores son decisivos en la motivación de los atletas jóvenes, en el compromiso deportivo y en el disfrute de las actividades deportivas (p.e., Duda y Balaguer, 2007; Duda y Appleton, 2016; Duda, Appleton, Stebbings, y Balaguer, 2018).

Respecto al clima motivacional creado por los entrenadores, Duda (2013), propuso un modelo multidimensional y jerárquico del clima motivacional en el contexto deportivo tomando como base la Teoría de las Metas de Logro (Achievement Goal Theory, AGT, Ames, 1992, Nicholls, 1989) y la Teoría de la Autodeterminación (Self-determination Theory, SDT, Ryan y Deci, 2017), en el que se establece que el clima puede ser más o menos empowering o disempowering. El clima motivacional empowering (funcionamiento positivo) se da cuando los entrenadores fomentan una alta implicación en la tarea (valoran el dominio en la tarea, aprendizaje, 


\section{Gutiérrez-García, P., López-Walle, J., Tomás, I., Tristán, J., y Balaguer, I.}

esfuerzo, todos tienen un lugar importante en el equipo, cooperación), un alto apoyo a la autonomía (ofrecen apoyo, flexibilidad, motivación a través del interés, y elección siempre que sea posible) y alto apoyo social (atmósfera de comprensión $\mathrm{y}$ confianza). Mientras que el clima disempowering (funcionamiento negativo) se caracteriza porque los entrenadores fomentan una alta implicación en el ego (comparación social, rivalidad interpersonal, castigo de los errores, evaluación pública) y utilizan un alto estilo controlador (intimidación, uso de técnicas coercitivas, excesivo control personal) en sus actitudes y conductas (Duda, 2013; Duda et al., 2013).

La Teoría de la Autodeterminación (SDT, Ryan y Deci, 2017), es una teoría motivacional que es útil para comprender la motivación de los individuos, sus causas y sus consecuencias. La SDT propone que los tipos de motivación tendrán consecuencias positivas o negativas para las personas dependiendo de las razones por las cuales participan en la actividad. Esta teoría ha identificado diferentes tipos de motivación. La motivación intrínseca se considera el prototipo de motivación autónoma y de las actividades autodeterminadas; los atletas intrínsecamente motivados participan en su deporte por el disfrute, interés y satisfacción de la actividad en sí misma. $L a$ regulación integrada se da cuando la conducta es coherente con otros valores y necesidades de los deportistas. La regulación identificada se produce cuando los atletas juzgan el comportamiento como importante, y aunque éste está regulado y autodeterminado por ellos, todavía se practica por razones extrínsecas, por ejemplo, beneficios personales. La regulación introyectada representa las conductas que están comenzando a internalizarse, pero que no son verdaderamente autodeterminadas ya que la fuerza externa solo es reemplazada por la fuerza de control interno, por ejemplo, las acciones se realizan para no sentirse culpables. La regulación externa se refiere a aquellos comportamientos que están controlados por fuentes externas, tales como materiales de apoyo u obligaciones impuestas por otros. Finalmente, la no motivación se caracteriza por la falta de razones para realizar las actividades, llevando a las personas a devaluar la actividad y / o a sentirse incapaces de realizarlas (Deci y Ryan, 1985). Además, los tipos de motivación se agrupan a nivel amplio en motivación autónoma y controlada (Deci y
Ryan, 2008). La motivación autónoma se caracteriza por comportarse con un sentido pleno de volición y elección, mientras que la motivación controlada se caracteriza porque las personas sienten que actúan de forma controlada, es decir, debido a las presiones generadas por el contexto social (Pelletier y Sarrazin, 2007; Ródenas, 2015).

En el contexto de los deportes, estar motivado de manera autónoma se asocia con resultados deseables, como la diversión en el deporte (Balaguer, Castillo, y Duda, 2008), la autoestima (Cantú-Berrueto et al., 2016) y la salud y bienestar (Ryan y Deci, 2002). Mientras que tener una motivación controlada conduce a la enfermedad, incomodidad o abandono de una actividad (Cantú-Berrueto et al., 2016; Pineda-Espejel, Alarcón, López-Ruiz, Trejo, y Chávez, 2016). En el estudio se pretende probar que, cuando el deportista actúa con autonomía, el deporte se convierte en una práctica positiva para ellos, favoreciendo su diversión.

En diversas investigaciones (Balaguer et al., 2015; López-Walle et al., 2011; Reigal, Crespillo, Morillo, y Hernández-Mendo, 2018) se ha demostrado que cuando los jugadores perciben que los climas motivacionales que crean sus entrenadores tienen alto grado de implicación en la tarea y bajo de implicación en el ego, estarán favoreciendo la calidad de la motivación de sus jugadores.

Específicamente, Duda y colaboradores (2018) defienden que los climas empowering y disempowering en el deporte juvenil, pueden influir en la motivación de los deportistas, así como en la calidad del compromiso deportivo y desempeñar un papel para facilitar o dificultar la participación sostenida en el deporte.

Los climas motivacionales empowering o disempowering han sido asociados con las regulaciones motivacionales (Castillo, López-Walle, Tomás, y Balaguer, 2017; Fenton, Duda, Appleton, y Barret, 2017; López-Walle et al., 2018), la motivación autónoma (Fenton et al., 2017), la motivación autodeterminada (Castillo et al., 2017) e indicadores de bienestar, tales como la diversión (Appleton y Duda, 2016; Duda et al., 2018; Fenton et al., 2017; Krommidas et al., 2016). 


\section{Clima empowering, motivación autónoma y diversión en pitchers de béisbol}

En un estudio reciente (Fenton et al., 2017) se ofrece evidencia empírica sobre la interrelación positiva que se establece entre la percepción del clima empowering con la motivación autónoma y de esta última con la diversión de jóvenes atletas, e incluso, se mostró en este estudio que la percepción del clima empowering generado por el entrenador tiene un efecto indirecto positivo sobre el disfrute a través de la motivación autónoma.

En su estudio, Barbosa-Luna et al. (2017) hipotetizaron que la motivación autodeterminada actuaría como mediadora de la relación entre los climas motivacionales generados por el entrenador a nivel de equipo e indicadores de bienestar. Los resultados ofrecieron apoyo al papel mediador que desempeña la motivación autodeterminada en la relación indicada a nivel del equipo (nivel entre). Se encontró que la percepción del clima de implicación en la tarea se relacionaba positivamente con la motivación autodeterminada, mientras que el clima de implicación en el ego se relacionaba negativamente con la motivación autodeterminada. En general, la revisión de la literatura indica que tanto la motivación autónoma como la motivación controlada median la relación entre los climas motivacionales y los indicadores de bienestar.

Hasta ahora, solo en algunos estudios como los de Marcinko (2015) y López -Walle et al. (2018) se ha puesto a prueba el papel moderador de la motivación. Por ejemplo, Marcinko (2015) encontró que los motivos autónomos moderan la relación entre componentes del bienestar subjetivo y la salud física. En otro estudio realizado en el contexto deportivo (López-Walle et al., 2018) la motivaciónn autónoma modera la relación entre el clima empowering y el burnout. Sin embargo, no conocemos ningún estudio precedente que analice el papel moderador de la motivación autónoma entre un clima motivacional positivo (p.e., empowering) y un indicador de bienestar (p.e., diversión). Se asume que cuando el comportamiento de las personas está motivado de manera autónoma, significa que una persona se comporta de acuerdo con sus propios deseos y una parte auténtica de sí misma. Por tal motivo, el objetivo de este trabajo ha sido poner a prueba el rol moderador de la motivación autónoma en la relación entre la percepción del clima empowering generado por el entrenador y la diversión de los pitchers de béisbol. Por lo tanto, se formularon las siguientes hipótesis:

Hipótesis 1: La percepción del clima empowering presentará una relación positiva y significativa con la diversión de los pitchers.

Hipótesis 2: La motivación autónoma moderará la relación entre la percepción del clima empowering generado por el entrenador y la diversión de los pitchers.

\section{MATERIAL Y MÉTODOS}

\section{Diseño}

El estudio es de tipo no experimental transversal con un alcance descriptivo, correlacional-causal.

\section{Participantes}

La muestra de este estudio está compuesta por un total de 97 jóvenes pitchers de entre 13 y 16 años, con una edad promedio de 14.12 años $(D T=1.09)$, pertenecientes a 39 equipos de las diferentes ligas de béisbol de Hermosillo, Sonora, México. Los pitchers cuentan con 3.25 años de experiencia $(D T=1.02)$, el promedio de horas de entrenamiento es de 2.07 horas diarias $(D T=0.63)$, el número de días por semana que entrenan es de 2.24 días $(D T=0.76)$, mientras que el número promedio de años con el mismo entrenador fue de 1.55 años $(D T=0.75)$.

\section{Instrumentos}

Para evaluar el clima motivacional empowering se utilizó el Cuestionario de Clima Motivacional Empowering y Disempowering (EDMCQ-C, Empowering and Disempowering Motivational Climate Questionnaire-Coach, Appleton, Ntoumanis, Quested, Viladrich, y Duda, 2016) validado al castellano (Balaguer, Castillo y Tomás, en revisión), el cual se adaptó para pitchers de béisbol en el contexto mexicano. El cuestionario evalúa la percepción de dos dimensiones: clima motivacional empowering y disempowering creado por el entrenador. La dimensión de clima empowering está compuesta por 17 ítems, y se obtiene como el promedio de 3 factores: implicación en la tarea $(9$ ítems), apoyo a la autonomía (5 ítems) y apoyo social (3 ítems). El cuestionario está encabezado por el siguiente enunciado: "En mi equipo de béisbol...". La escala de respuesta es tipo Likert con cinco 


\section{Gutiérrez-García, P., López-Walle, J., Tomás, I., Tristán, J., y Balaguer, I.}

alternativas de respuesta $(1=$ muy en desacuerdo a 5 $=$ muy de acuerdo). Estudios previos ofrecen evidencia empírica que apoya la consistencia interna de la dimensión empowering (Appleton y Duda, 2016; Appleton et al., 2016; Balaguer et al., en revisión; Castillo et al., 2017).

Para evaluar la motivación autónoma, se utilizó la versión mexicana de la Escala de Motivación Deportiva (SMS-II, Sport Motivation Scale - II, Pelletier, Rocchi, Vallerand, Deci, y Ryan, 2013; Barbosa-Luna et al., 2017) la cual se adaptó para pitchers de béisbol. La escala consta de 18 ítems, que representan 6 regulaciones motivacionales (intrínseca, integrada, identificada, introyectada, externa, y no motivación), cada una de ellas medida con 3 ítems. Los lanzadores responden a través de una escala de respuesta de tipo Likert que oscila desde (1) Muy en desacuerdo a (7) Totalmente de acuerdo. Para evaluar la motivación autónoma, se consideró el promedio de los ítems de regulación intrínseca, regulación integrada y regulación identificada. Esta clasificación ha sido adoptada en diversos estudios (Barbosa-Luna et al., 2017; Lonsdale, Hodge, Hargreaves, y Ng, 2014; PinedaEspejel et al., 2016). En el contexto de los deportes, investigaciones previas apoyan la validez y fiabilidad de esta escala (Balaguer, Castillo, y Duda; 2007; Barbosa-Luna et al., 2017; Lonsdale et al., 2014; Pelletier et al., 2013; Pineda-Espejel et al., 2016).

Para evaluar la diversión en la práctica deportiva, los pitchers respondieron a la escala de Satisfacción Intrínseca en el Deporte (SSI, Intrinsic SatisfactionSport Scale, Duda y Nicholls, 1992) en su versión española (Castillo, Balaguer, y Duda, 2002), la cual se adaptó al pitcher en el contexto mexicano. El cuestionario consta de 7 ítems divididos en dos escalas: diversión ( 5 ítems) y aburrimiento ( 2 ítems). La escala de respuesta es de tipo Likert de 5 puntos que oscila desde (1) Totalmente en desacuerdo a (5) Totalmente de acuerdo. En este estudio se utilizaron solamente los ítems de la escala de diversión. En el contexto deportivo, investigaciones previas han apoyado la validez y fiabilidad de esta escala (SSI) (Castillo et al., 2002; Castillo, Balaguer, Duda, y García-Merita, 2004; Duda y Nicholls, 1992).

\section{Procedimiento}

Se solicitaron los permisos previos para realizar el estudio a la Asociación Estatal de béisbol de Sonora, y posterior, para la recogida de datos a las directivas de las diferentes Ligas de béisbol de Hermosillo, Sonora. Además, respetando los protocolos éticos y considerando que los participantes eran menores de edad, se solicitó a los padres de los deportistas la firma del Consentimiento Informado de Participación en el estudio, previo a la aplicación de los cuestionarios, y se informó que las respuestas y el manejo de los datos se mantendrían de forma confidencial. La recogida de datos tuvo lugar en sesiones previas a los entrenamientos. Se explicó brevemente a los deportistas el objetivo del estudio y se les entregó el cuadernillo de aplicación. Al menos un investigador estuvo presente en todo momento y tuvo el apoyo de estudiantes de la carrera de Licenciatura en Entrenamiento Deportivo. El tiempo promedio de la aplicación de la batería de cuestionarios fue de 20 minutos.

\section{Análisis estadístico}

En primer lugar y dado que se adaptaron los instrumentos a los pitcher de béisbol, se recogieron evidencias de validez (análisis factorial confirmatorio, AFC) y fiabilidad (coeficiente alpha de Cronbach, y coeficiente rho o de fiabilidad compuesta) de las escalas de medida. Valores del coeficiente de fiabilidad compuesta iguales o mayores a .70 indican adecuada fiabilidad (Raykov, 2001). Respecto al AFC, se pusieron a prueba modelos unifactoriales por separado para cada una de las escalas utilizadas en el estudio. Asimismo se calcularon los estadísticos descriptivos (media y desviación típica) y correlaciones entre las variables implicadas en el estudio.

El modelo puesto a prueba analizó el papel moderador de la forma de motivación autónoma en la relación entre la percepción del clima empowering creado por el entrenador y la diversión en la práctica por parte de los pitchers. Para poner a prueba dicho modelo se utilizó la macro PROCESS (Hayes, 2013) para SPSS. Para probar los efectos condicionales o efectos de interacción, se utilizaron intervalos de confianza bootstrap, ajustados por sesgo, con 10,000 repeticiones y nivel de confianza del 95\%. Esta macro permite realizar un análisis de regresión múltiple que estima los coeficientes de regresión no estandarizados para las variables del modelo. 


\section{Clima empowering, motivación autónoma y diversión en pitchers de béisbol}

Además, ofrece el efecto condicional de la variable independiente (clima empowering) sobre la dependiente (diversión) para diferentes valores del moderador (motivación autónoma), utilizando la técnica de Johnson-Neyman (J-N), y permite obtener gráficos de interacción que representan el rol de la variable moderadora en la relación entre la variable independiente y la dependiente (Hayes, 2013).

\section{RESULTADOS}

Análisis factorial confirmatorio de las escalas y fiabilidad

Como se ha comentado anteriormente, el primer paso fue analizar la validez factorial y fiabilidad de las escalas de medida. En la Tabla 1 se observan los índices de bondad de ajuste de los modelos unifactoriales puestos a prueba para cada una de las escalas. Respecto a clima empowering, el modelo unifactorial mostró índices de bondad de ajuste adecuados $\left(\chi^{2} / g l=1.83\right.$, CFI $=.90$, TLI $=.90$, RMSEA $=.09)$. Los índices de ajuste de los modelos unifactoriales para la escala de motivación autónoma $\left(\chi^{2} / g l=3.39, \mathrm{CFI}=.91, \mathrm{TLI}=.90, \mathrm{RMSEA}=.16\right) \mathrm{y}$ para la escala de diversión $\left(\chi^{2} / g l=3.07, \mathrm{CFI}=.94\right.$, TLI $=.90$, RMSEA $=.15)$ fueron satisfactorios, excepto por el RMSEA que presentó valores por encima de .10 en ambos casos.

La consistencia interna de todas las escalas fue satisfactoria (ver Tabla 1), presentando valores de alfa de Cronbach igual o por encima de .78. Respecto a la fiabilidad compuesta, estuvo por encima de .70 para todas las escalas. Concretamente, presentó valores entre .83 y .94 .

\section{Estadísticos descriptivos y correlaciones entre las variables del estudio}

La Tabla 2 muestra el rango de respuesta, media, desviación típica (DT) y correlaciones existentes entre las variables del estudio. Los análisis indicaron una relación positiva y significativa entre el clima empowering y la diversión (tal como se planteaba en la Hipótesis 1), lo cual se confirma con el análisis de correlación $(r=.33, p<.01)$. Además, la motivación autónoma presentó relación positiva y significativa tanto con el clima empowering, como con la diversión.

\section{Modelo de regresión moderada}

Los resultados del modelo de regresión moderada (ver Tabla 3) indicaron que el término de interacción entre el clima empowering y la motivación autónoma fue estadísticamente significativo y que el intervalo de confianza (IC) bootstrap para dicho efecto no incluía el valor cero $(\mathrm{B}=-.15, p<.05$, IC 95\% $=[-$ $.29,-.02])$, ofreciendo apoyo empírico para la Hipótesis 2.

Además, la motivación autónoma presentó una relación positiva y significativa $(\mathrm{B}=.33, p<.001)$ con la diversión. El modelo de regresión explicó el $31 \%$ de la varianza de la diversión de los pitchers $\left[R^{2}\right.$ $=.31, F(3,93)=13.70, p<.001]$. Además, el cambio en el $R^{2}$ con la adición del efecto moderador de la motivación autónoma (MA) fue significativo $\left[\Delta R^{2}=.03, \mathrm{~F}(1,93)=4.57, p<.05\right]$, indicando que la interacción entre el clima empowering y la motivación autónoma explicaba un 3\% adicional de la varianza de la diversión, al ya explicado por el clima empowering y la motivación autónoma. Los resultados indicaron que la motivación autónoma modera la relación entre el clima empowering y la diversión.

En la Figura 1 se ofrece la representación gráfica del modelo de regresión moderada que muestra la interacción del clima empowering y la motivación autónoma como predictores de la diversión.

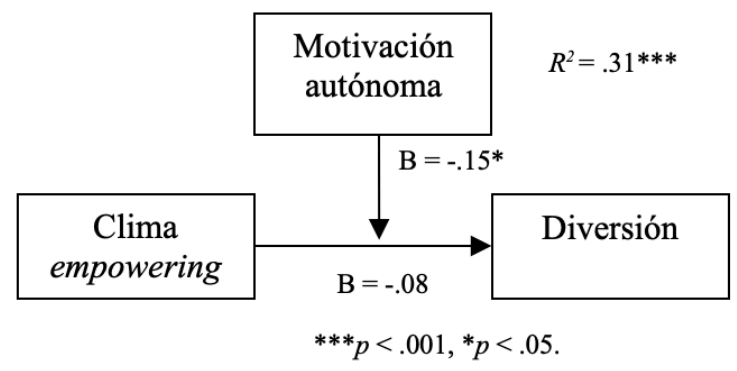

Figura 1. Modelo de regresión moderada: interacción del clima empowering y la motivación autónoma como predictores de la diversión. 


\section{Gutiérrez-García, P., López-Walle, J., Tomás, I., Tristán, J., y Balaguer, I.}

Tabla 1.

Resultados del análisis factorial confirmatorio y fiabilidad de las escalas.

\begin{tabular}{lllllllll}
\hline & $\chi^{2}$ & $g l$ & $\chi^{2} / g l$ & CFI & TLI & RMSEA & $\alpha$ & CR \\
\hline 1. Clima empowering & .22 & 116 & 1.83 & .90 & .90 & .09 & .93 & .94 \\
2. Motivación autónoma & .81 & 24 & 3.39 & .91 & .90 & .16 & .91 & .83 \\
3. Diversión & .12 & 4 & 3.07 & .94 & .90 & .15 & .78 & .91 \\
\hline
\end{tabular}

$\alpha$ : Alfa de Cronbach. CR: Fiabilidad compuesta.

Tabla 2 .

Estadísticos descriptivos y correlaciones bivariadas entre las variables del estudio.

\begin{tabular}{lllllll}
\hline & Rango & $M$ & $D T$ & 1 & 2 & 3 \\
\hline 1. Clima empowering & $1-5$ & 4.40 & 0.52 & -- & & \\
2. Motivación autónoma & $1-7$ & 6.10 & 1.06 & $.49^{* *}$ & -- & \\
3. Diversión & $1-5$ & 4.20 & 0.82 & $.33^{* *}$ & $.53^{* *}$ & -- \\
\hline
\end{tabular}

$* * p<.01$

Tabla 3.

Modelo de regresión moderada: Interacción entre el clima empowering y la motivación autónoma como predictores de la diversión.

Variable dependiente: Diversión

\begin{tabular}{lllll}
\hline & $\mathrm{B}$ & $t$ & LI IC & LS IC \\
\hline Predictor & & & & \\
\hline Motivación autónoma & $.33^{* * *}$ & 4.15 & .17 & .49 \\
Clima empowering & -.08 & -.45 & -.44 & .28 \\
Interacción & $-.15^{*}$ & -2.14 & -.29 & -.02 \\
\hline
\end{tabular}

$\mathrm{R}^{2}=.31 * * *$

$\Delta \mathrm{R}^{2}=.03 * *$

Nota. $\mathrm{B}=$ coeficiente beta no estandarizado, LI IC = límite inferior de intervalo de confianza, LS IC = límite superior del intervalo de confianza, $\Delta \mathrm{R}^{2}=$ incremento en $\mathrm{R}^{2}$ debido a la interacción.

$* * * p<.001,{ }^{* *} p<.01,{ }^{*} p<.05$.

Siguiendo el procedimiento propuesto por Aiken y West (1991), para interpretar el efecto de interacción formulado en la Hipótesis 2, se estimaron y representaron gráficamente las pendientes simples de la relación entre clima empowering y diversión, para diferentes valores del moderador (una DT por encima, una DT por debajo, 2 DT por debajo, y 3.5 DT por debajo de la media muestral en motivación autónoma), y se representaron gráficamente las correspondientes líneas de regresión (ver Figura 2). Hay que tener en cuenta, que la motivación autónoma de los sujetos de la muestra fue muy elevada, ya que presentó un valor promedio de $6.10(D T=1.06)$ en una escala donde el valor máximo es el 7 , lo cual condicionó la elección de los valores del moderador para ser representados gráficamente. La figura muestra que cuando la motivación autónoma fue muy alta $(\mathrm{MA}=7)$ considerando el rango de valores de la escala (el valor +1 DT fue reemplazado por 7, el valor máximo de la escala, ya que el valor media +1 DT estaba por encima del rango posible de la escala de motivación autónoma), el disfrute fue también elevado y no hubo relación significativa entre el clima empowering y la diversión $(\mathrm{B}=-.22, p>.05)$. Cuando la motivación autónoma estuvo 1 DT por debajo de la media de la muestra $(\mathrm{MA}=5.04$, indicando valores altos en motivación autónoma según el rango de la escala), el nivel de disfrute fue menor, y la relación entre clima empowering y diversión tampoco fue significativa $(\mathrm{B}=.08, p$ $>$.05). Cuando la motivación autónoma estuvo 2 DT por debajo de la media de la muestra $(\mathrm{MA}=3.98$, indicando valores medios en motivación autónoma según el rango de la escala), el nivel de disfrute 


\section{Clima empowering, motivación autónoma y diversión en pitchers de béisbol}

también fue menor, y la relación entre clima empowering y diversión tampoco fue significativa (B $=.24, p>.05)$. Sin embargo, cuando la motivación autónoma fue baja según el rango de respuesta de la escala $(\mathrm{MA}=2.40$, que se correspondería con un valor de -3.5 DT por debajo de la media de la muestra del estudio); la relación entre clima empowering y diversión fue positiva y significativa $(\mathrm{B}=.48, p<.05)$.

Finalmente, con la utilidad de la técnica estadística Johnson-Neyman (J-N), que permite obtener un rango mayor de valores de la variable moderadora, se pudo observar el efecto de la motivación autónoma sobre la relación entre el clima empowering y la diversión, para todo el rango de valores posibles de la motivación autónoma. Los resultados indicaron que para valores por debajo de 2.50 en motivación autónoma (el 3.1\% de la muestra), la relación entre el clima empowering y la diversión fue positiva $\mathrm{y}$ estadísticamente significativa $(M=2.50, \mathrm{~B}=.47, p$ $<.05)$.

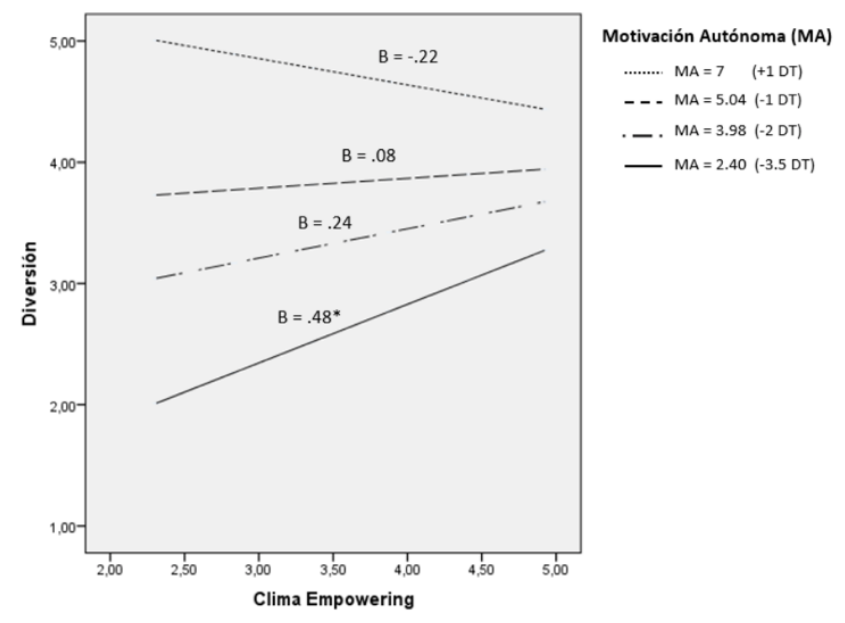

Figura 2. Gráfica del efecto condicional de la motivación autónoma en la relación entre clima empowering y diversión.

\section{DISCUSIÓN}

El propósito de este estudio fue poner a prueba el papel moderador de la forma de motivación autónoma entre la percepción del clima empowering creado por el entrenador y la diversión en la práctica por parte de pitchers de béisbol. El papel moderador de la motivación se ha estudiado entre otros, en el contexto de la psicología organizacional, la psicología educativa y la psicología del deporte que es el contexto en el que se llevó a cabo esta investigación.

En primera instancia, se confirma que la percepción del clima empowering creado por el entrenador se relaciona positivamente con indicadores de bienestar como predice la teoría de la SDT (Duda et al., 2018) y en nuestro caso específicamente con la diversión, lo cual ha recibido apoyo empírico también en trabajos previos en el contexto deportivo (Fenton et al., 2017).

Existe bastante evidencia empírica sobre las relaciones positivas que se establecen entre las subdimensiones del clima empowering (clima de implicación en la tarea, estilo de apoyo a la autonomía y apoyo social) con la motivación autodeterminada y con la diversión (Duda y Balaguer, 2007; Roberts, 2012), pero poco se sabe todavía sobre el grado en el que la magnitud de la relación entre el clima empowering y la diversión depende de la motivación autónoma. Los hallazgos del presente estudio ofrecen apoyo a la hipótesis 2 informado que la motivación autónoma modera la relación entre la percepción del clima empowering generado por el entrenador y la diversión de los pitchers.

En el estudio se observa que el clima empowering va a ser sobre todo importante para aquellos deportistas (pitchers) que tengan poca motivación autónoma. Parece ser que los que tienen alta motivación autónoma, disfrutan mucho, independientemente de la percepción que tengan del grado del clima empowering creado por el entrenador. Aquellos deportistas (pitchers) que tienen baja motivación autónoma, se ven altamente beneficiados cuando perciben que sus entrenadores fomentan un clima empowering, ya que esto hace que su diversión aumente (hay relación positiva y significativa entre clima empowering y diversión). Estos hallazgos refuerzan la importancia que tiene que los entrenadores aprendan a potenciar el desarrollo positivo de los deportistas a través de la creación de un clima alto en implicación en la tarea, en apoyo a la autonomía y en la creación de una atmosfera de comprensión y confianza en los jóvenes. La percepción de este tipo de climas favorece que se produzca una participación más entusiasta $\mathrm{y}$ satisfactoria en los entrenamientos, lo que puede 


\section{Gutiérrez-García, P., López-Walle, J., Tomás, I., Tristán, J., y Balaguer, I.}

contribuir al desarrollo de un mayor compromiso en la práctica del béisbol.

Los resultados de este estudio nos indican que es importante que el entrenador diseñe entrenamientos en los que se valore el progreso y el esfuerzo ya que eso ayudará a que los deportistas se muevan por motivos intrínsecos. La investigación ha informado consistentemente que cuando se valora el resultado, en lugar del progreso, se dificulta el desarrollo de la motivación autónoma y se potencia la motivación controlada (Ryan y Deci, 2017). Los estudios basados en las teorías de las metas de logro y la teoría de la autodeterminación han demostrado que cuando los entrenadores favorecen la motivación intrínseca en deportistas de diferentes niveles (p.ej., deporte de recreación, jóvenes talentos) es más probable que estos se diviertan y consigan un optimo desarrollo (Duda y Balaguer, 2007; Ryan y Deci, 2017).

Dentro de las limitaciones del estudio se puede señalar el número de sujetos, ya que se consideró únicamente una posición del béisbol, dada la complejidad de la misma, como futura línea de investigacion se sugiere aumentar la muestra y el rango de edad de los lanzadores. Además sería interesante analizar cómo se comportan estos indicadores psicológicos en diferentes momentos de la preparación durante la temporada en la que participan los pitcher, no sólo en entrenamiento sino además en juegos y en competencia.

\section{CONCLUSIONES}

Como conclusiones, se puede señalar que el estudio realizado confirma la importancia de que el entrenador se preocupe por generar ambientes empoderadores que promuevan una motivación autónoma para que el lanzador se divierta. Cuando el entrenador crea climas de empoderamiento va a fomentar ambientes caracterizados por hacer sentir a los pitchers que actúan con autonomía y libertad en la toma de decisiones, lo que favorecerá el disfrute en las actividades del entrenamiento y de la competicion.

Nos queda por explicar lo que sucede con ese porcentaje de sujetos que tienen una motivación autónoma baja a pesar de percibir un clima de empoderamiento en sus equipos. Futuros estudios deberían exploran si existen otras variables, como por ejemplo variables de personalidad que neutralicen esta relación entre el clima empowering y la motivación autónoma. También habría que averiguar el tiempo que necesitarían estar estos sujetos que tienen baja motivación autónoma en un clima de empoderamiento para que esta pudiese incrementarse.

\section{APLICACIONES PRÁCTICAS}

Desde un punto de vista aplicado, los hallazgos del presente estudio subrayan la importancia de que los entrenadores refuercen la motivación autónoma y creen entornos que fomenten la diversión de los jóvenes en los entrenamientos, garantizando que éstos se sientan atendidos, respetados y conectados a los demás. También nos indican que los entrenadores tienen que procurar que los deportistas sientan que tienen voluntad de elección ya que con ello también se promoverá su motivación autónoma y su implicación en la tarea.

\section{AGRADECIMIENTOS}

Esta investigación ha sido parcialmente financiada por el PFCE-2017, el CONACYT y la UANL. Este estudio se ha realizado en conjunto con doctores de la Facultad de Organización Deportiva, Universidad Autónoma de Nuevo León, México, y revisado en la parte teórica y metodológica por expertos de la Universidad de Valencia, España.

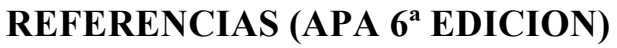

1. Aiken, L. S., y West, S. G. (1991). Multiple regression: Testing and interpreting interactions. Newbury Park, CA: Sage.

2. Ames, C. (1992). Classrooms: Goals, structures, and student motivation. Journal of Educational Psychology, 84(3), 261-271. http://dx.doi.org/10.1037/0022-0663.84.3.261

3. Appleton, P. R., y Duda, J. L. (2016). Examining the interactive effects of coachcreated empowering and disempowering climate dimensions on athletes' health and functioning. Psychology of Sport and Exercise, 26 ,

$61-70$. https://doi.org/10.1016/j.psychsport.2016.06.00 7 


\section{Clima empowering, motivación autónoma y diversión en pitchers de béisbol}

4. Appleton, P. R., Ntoumanis, N., Quested, E., Viladrich, C., y Duda, J. L. (2016). Initial validation of the coach-created Empowering and Disempowering Motivational Climate Questionnaire (EDMCQ-C). Psychology of Sport and Exercise, 22, 53-65. https://doi.org/10.1016/j.psychsport.2015.05.00 8

5. Balaguer, I., Castillo, I., y Duda, J. L. (2007). Propiedades psicométricas de la Escala de Motivación Deportiva en deportistas españoles. Revista Mexicana de Psicología, 24, 197- 207.

6. Balaguer, I., Castillo, I., y Duda, J. L. (2008). Apoyo a la autonomía, satisfacción de las necesidades, motivación y bienestar en deportistas de competición: Un análisis de la teoría de la autodeterminación. Revista de Psicología del Deporte, 17(1), 123-139.

7. Balaguer, I., Castillo, I., Ródenas, L., Fabra, P., y Duda, J. L. (2015). Los entrenadores como promotores de la cohesión del equipo. Cuadernos de Psicología del Deporte, 15(1), 233-242.

8. Barbosa-Luna, A. E., Tristán, J. L., Tomás, I., González, A., y López-Walle, J. M. (2017). Climas motivacionales, motivación autodeterminada, afectos y burnout en deportistas: enfoque multinivel. Acción Psicológica, 14(1), 105-117. http://dx.doi.org/10.5944/ap.14.1.19266

9. Cantú-Berrueto, A., López-Walle, J., Castillo, I., Tristán, J., Álvarez, O., y Balaguer, I. (2016). The controlling interpersonal style, types of motivation, self-esteem and burnout in Mexican athletes. Journal of Sports \& Exercise Psychology, 38, S169.

10. Castillo, I, Balaguer, I., y Duda, J. L. (2002). Las perspectivas de meta de los adolescentes en el contexto deportivo. Psicothema, 14(2), 280287.

11. Castillo, I., Balaguer, I., Duda, J., y GarcíaMerita, M. (2004). Factores psicosociales asociados con la participación deportiva en la adolescencia. Revista Latinoamericana de Psicología, 36(3), 505-515.

12. Castillo, N., López-Walle, J. M., Tomás, I., y Balaguer, I. (2017). Relación del clima empowering con la motivación autodeterminada a través de la satisfacción de las necesidades psicológicas básicas. Revista de Psicología del Deporte, 26(3), 33-39.

13. Deci, E. L. y Ryan, R. M. (1985). Intrinsic motivation and self-determination in human behavior. New York, N.Y.: Plenum Press. http://dx.doi.org/10.1007/978-1-4899-2271-7

14. Deci, E. L. y Ryan, R. M. (2008). Selfdetermination theory: A macrotheory of human motivation, development, and health. Canadian Psychology/Psychologie Canadienne, 49(3), 182-185. http://dx.doi.org/10.1037/a0012801

15. Duda, J. L. (2013). The conceptual and empirical foundations of Empowering Coaching $^{\mathrm{TM}}$ : Setting the stage for the PAPA project. International Journal of Sport and Exercise Psychology, 11(4), 311-318. https://doi.org/10.1080/1612197X.2013.839414

16. Duda, J. L., y Appleton, P. R. (2016). Empowering and Disempowering Coaching Climates: Conceptualization, Measurement Considerations, and Intervention Implications. En M. Raab, P. Wylleman, R. Seiler, A-M. Elbe, y A. Harzigeorgiadis, A. (Eds), Sport and Exercise Psychology Research: From theory to practice (pp. 374-390). New York, NY: Academic. https://doi.org/10.1016/B978-0-12803634-1.00017-0

17. Duda, J. L., y Balaguer, I. (2007). Coachcreated motivational climate. En S. Jowette y D. Lavallee (Eds.), Social psychology in sport, (pp. 117-130). Champaign, IL.: Human Kinetics.

18. Duda, J. L., Appleton, P. R., Stebbings, J., y Balaguer, I. (2018). Towards more empowering and less disempowering environments in youth sport. En C. J. Knight, C. G. Harwood, y D. Gould (Ed.), Sport Psychology for Young Athletes (pp. 81-93). New York, NY: Routledge. http://dx.doi.org/10.4324/9781315545202-8

19. Duda, J. L. y Nicholls, J. G. (1992). Dimensions of achievement motivation in schoolwork and sport. Journal of Educational Psychology, 84(3), 290. https://doi.org/10.1037/0022-0663.84.3.290 


\section{Gutiérrez-García, P., López-Walle, J., Tomás, I., Tristán, J., y Balaguer, I.}

20. Duda, J. L., Quested, E., Haug, E., Samdal, O., Wold, B., Balaguer, I., ... y Cruz, J. (2013). Promoting Adolescent health through an intervention aimed at improving the quality of their participation in Physical Activity (PAPA): Background to the project and main trial protocol. International Journal of Sport and Exercise Psychology, 11(4), 319-327. https://doi.org/10.1080/1612197X.2013.839413

21. Ealo, J. (1984). Béisbol. La Habana, Cuba: Pueblo y educación.

22. Fenton, S. A., Duda, J. L., Appleton, P. R., y Barrett, T. G. (2017). Empowering youth sport environments: implications for daily moderateto-vigorous physical activity and adiposity. Journal of Sport and Health Science, 6(4), 423433.

http://dx.doi.org/10.1016/j.jshs.2016.03.006

23. González, J., Gotera E., y Cobos, I. (2009). Análisis descriptivo de variables cinemáticas de la acción técnica del pitcheo en béisbol. Omnia, 15(3), 44-57.

24. Hayes, A. F. (2013). Introduction to mediation, moderation, and conditional process analysis: $A$ regression-based approach. New York, NY, US: The Guilford Press.

25. Krommidas, C., Galanis, E., Papaioannou, A., Tzioumakis, G., Keramidas, P., y Digelidis, N. (2016). The relationship of empowering and disempowering coaching climate with enjoyment and quality of life variables in greek youth soccer. Inquiries in Sport \& Physical Education, 14(2), 19-35.

26. Lonsdale, C., Hodge, K., Hargreaves, E. A., y Ng, J. Y. (2014). Comparing sport motivation scales: A response to Pelletier et al. Psychology of Sport and Exercise, 15(5), 446-452. https://doi.org/10.1016/j.psychsport.2014.03.00 6

27. López-Walle, J., Balaguer, I., Castillo, I., y Tristán, J. (2011). Clima motivacional percibido, motivación autodeterminada $\mathrm{y}$ autoestima en jóvenes deportistas mexicanos. Revista de Psicología del Deporte, 20(1), 209222 .

28. López-Walle, J., Tristán, J., Flores, M., Pérez, M., Atienza, F., y Balaguer, I. (2018).
Perceived coach empowering climate and burnout: The moderator role of athletes autonomous motivation. En M. Murphy, C. Boreham, G. De Vito, y E. Tsolakidis (Eds.), Book of Abstracts of the $23^{\text {rd }}$ Annual Congress of the European College of Sport Science (p. 266). Alemania: SportTools.

29. Marcinko, I. (2015). The Moderating Role of Autonomous Motivation on the Relationship between Subjective Well-Being and Physical Health. PLoS ONE 10(5), 1-17. http://dx.doi.org/10.1371/journal.pone.0126399

30. Nicholls, J. G. (1989). The competitive ethos and democratic education. Cambridge, MA: Harvard University Press.

31. Pelletier, L., Rocchi, M., Vallerand, R., Deci, E. L., y Ryan, R. M. (2013). Validation of the revised sport motivation scale (SMS-II). Psychology of Sport and Exercise, 14, 329-341. https://doi.org/10.1016/j.psychsport.2012.12.00 2

32. Pelletier, L. G., y Sarrazin, P. (2007). Measurement issues in self-determination theory and sport. En M. S. Hagger y N. L. D. Chatzisarantis (Eds.), Intrinsic Motivation and Self-determination in Exercise and Sport (pp. 143-152). Champaign, IL, US: Human Kinetics.

33. Pineda-Espejel, H., Alarcón, E., López-Ruiz, Z., Trejo, M., y Chávez, C. (2016). Propiedades psicométricas de la Escala de Motivación en el Deporte revisada (SMS-II) adaptada al español hablado en México. RICYDE: Revista Internacional de Ciencias del Deporte, 44(12), 107-120.

https://doi.org/10.5232/ricyde2016.04402

34. Raykov, T. (2001). Estimation of congeneric scale reliability using covariance structure analysis with nonlinear constraints. British Journal of Mathematical and Statistical Psychology, 54, 315-323. http://dx.doi.org/10.1348/000711001159582

35. Reigal, R.E.; Crespillo, M.; Morillo, J.P.; Hernández-Mendo, A. (2018). Apoyo a la autonomía, clima motivacional percibido y perfil psicológico deportivo en jugadores de 


\section{Clima empowering, motivación autónoma y diversión en pitchers de béisbol}

balonmano playa. Cuadernos de Psicología del Deporte, 18(3), 102-111.

36. Ríos, E. (2010). Comportamiento de indicadores fisiológicos con la ejecución de pruebas de terreno en beisbolistas juveniles nacionales (Tesis de maestría). Facultad de Ciencias Médicas, La Habana, Cuba.

37. Roberts, G. C. (2012). Motivation in sport and exercise from an achievement goal perspective. After 30 years, where are we? En G. C., Roberts y D. C. Treasure (Eds.). Advances in Motivation Sport and Exercise (pp. 5-58). Champaign, IL: Human Kinetics.

38. Ródenas, L. T. (2015). Clima motivacional, motivación y cohesión: un estudio en el fútbol base (Tesis doctoral). Universidad de Valencia, España.

39. Ryan, R. M. y Deci, E. L. (2017). Selfdetermination theory: Basic psychological needs in motivation, development, and wellness. New York: Guilford.

40. Ryan, R. M. y Deci, E. L. (2002). Overview of Self-Determination Theory: An organismic dialectical perspective. En E.L. Deci, y R.M. Ryan, (Eds.), Handbook of Self-Determination Research (pp. 3-33). Rochester: The University of Rochester Press. 